\title{
Performance Evaluation of IPv4 and IPv6 on Windows Vista and Linux Ubuntu
}

\author{
Shaneel Narayan (Member IEEE), Peng Shang, Na Fan \\ School of Computing and Information Technology \\ UNITEC New Zealand \\ Auckland \\ snarayan@unitec.ac.nz
}

\begin{abstract}
A small network of computing devices that started as ARPANET project in early 1980s is now a worldwide network of devices for billions of users. This global network, the Internet, has become an integral part of worldwide economy and life of individuals. Internet Protocol (IP) v4 is the basic building block of the Internet and has served well, but it has limitations that hinder its growth. The solution is IPv6, which addresses inherent problems of the earlier version. However, due to the increased overhead in IPv6 and its interaction with the operating system that hosts this communication protocol, there may be network performance issues. In this paper, two operating systems namely, Windows Vista and Linux Ubuntu are configured with the two versions of IP and empirically evaluated for performance difference. Performance related metrics like throughput, delay, jitter and CPU usage are empirically measured on a test-bed implementation. The results show that network performance depends not only on IP version and traffic type, but also on the choice of the operating system.
\end{abstract}

Keywords- TCP/IP, IPv4, IPv6, performance evaluation, Windows Vista, Ubuntu

\section{INTRODUCTION}

With organizations striving to continuously increase business, the Internet has become a necessary arsenal in the armory of any $21^{\text {st }}$ century businesses. Internet Protocol version 4 (IPv4) is the basic building block used to construct Internet infrastructure; however there are limitations in this technology. The major shortfall of IPv4 is that it has limited address space of approximately 4.3 billion $\left(2^{32}\right)$, but the real world limit falls far short of this theoretical cap due to the way addresses are administered globally [1]. Less than $16 \%$ addresses remain unallocated as of early 2008 and it is expected that by 2010 or 2011 IPv4 addresses will run out [2]. This problem was foreseen in the late 1990 and in response Internet Engineering Task Force (IETF) began work on the development of a new version of Internet Protocol, IPv6. This new version has lots of improvements including; increased address space from $2^{32}$ to $2^{128}$, enhanced security, enhanced end user benefits, mobility support and integrated Quality of Service (QoS). However, IPv6 data header is twice in size to that of its predecessor, implying that IPv6 has a higher overhead associated with it thus performance degradation. This additional over varies in size with the data payload size - for example, with no fragmentation of datagrams, a payload of 128 Bytes has performance difference of $10.3 \%$, and for payload of 1408 Bytes the difference is $1.3 \%$ approximately [3]. In this paper,
IPv4 and IPv6 performance has been compared on two operating systems namely, Windows Vista and Linux Ubuntu. Results obtained from this test bed analysis show that theoretical difference in performance of the two versions of IP is different to that on real implementations. Also IPv4 and IPv6 performance values are different for the two operating systems.

The rest of the paper is organized as follows: Section 2 discusses some of similar work undertaken by other researchers, Section 3 describes Internet Protocols mainly focusing on IPv6 header structure and Section 4 outlines the experimental setup used in this research. We present the results and discuss the findings in Section 5. Finally, the research is concluded in Section 6.

\section{RELATED WORK}

Research related to performance of IPv4 and IPv6 has been undertaken by a number of researchers. There are a number of threads in research related to this topic, however research specific to performance of protocols on operating systems based on test-bed experiments are outlined in Figure 1. Simulations and emulations can be employed as other means of testing, but test-bed experiments show that performance of IPv4 and IPv6 varies to different degrees depending on the

\begin{tabular}{|ll|}
\hline \multicolumn{1}{|c|}{ RESEARCHER(S) } & \multicolumn{1}{c|}{ OPERATING SYSTEM(S) } \\
Narayan, et al. (2008) [3] & $\begin{array}{l}\text { Windows 2003 and Windows XP (IPv4, } \\
\text { IPv6) }\end{array}$ \\
Visoottiviseth \& Bureenok (2008) [4] & $\begin{array}{l}\text { Windows 2003, Red Hat 9.0, FreeBSD } \\
5.3 \text { (IPv4, IPv6) }\end{array}$ \\
Mohamed, Buhari \& Saleem (2006) [5] & $\begin{array}{l}\text { Windows 2003, Redhat 9.0, FreeBSD 4.9 } \\
\text { (IPv6) }\end{array}$ \\
Ahuja \& Dendukuri (2006) [6] & Windows 2000 and Linux (IPv4, IPv6) \\
Zeadally, Wasseem \& Raicu (2004) [7] & $\begin{array}{l}\text { Windows 2000, Solaris 8 \& Redhat 7.3 } \\
\text { (IPv4, IPv6) }\end{array}$ \\
Zeadally \& Raicu (2003) [8] & Windows 2000 and Solaris 8 (IPv4, IPv6) \\
Anand (2000) [9] & Linux kernel 2.4.17 (IPv6) \\
Ettikan (2000) [10] & FreeBSD (KAME IPv6) \\
Ariga, et al. (2000) [11] & FreeBSD (KAME IPv6) \\
Xie (1999) [12] & Windows NT (MSR IPv6 Beta) \\
Draves, Mankin \& Zill (1998) [13] & Windows NT (MSR IPv6) \\
\hline
\end{tabular}

Figure 1: Research Related to Test-bed IP Performance Analysis

operating system it is implemented on. For example changing from IPv4 to IPv6 on Windows NT downgrades performance by $2 \%$ [13], FreeBSD performs slightly inferior [9], [10], [11], 
[12]. In [3] Windows XP and Server 2003 perform differently with the two protocols and performance varies with packet size.

In this research we continue analyzing performance of IPv4 and IPv6 on Windows Vista SP1 and Linux Ubuntu (kernel 2.6.24_21 generic). The novelty of this research is that analyses of these two operating system performances with IPv4/IPv6 are not present in literature.

\section{BACKGROUND}

IPv6 has been designed as an evolutionary step from IPv4 and it addresses problems inherent in IPv4, plus offers new opportunities for services that can be provided via computer networks. In [14], the author discusses these opportunities in detail, and at data packet level the difference between IPv4 and IPv6 is illustrated in Figure 2. Comparing the two, IPv6 (second picture) has larger space for addresses (expanded from 32 to 128bits); however the length of the header has not

\begin{tabular}{|c|c|c|c|c|c|c|c|}
\hline Version & Length & Senvice Type & Packet Length & Yesion Number & Prioity & \multicolumn{2}{|c|}{ Fbon Lasol } \\
\hline \multicolumn{3}{|c|}{ Identification } & 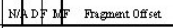 & \multicolumn{2}{|l|}{ Pashod Langhth } & 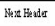 & ${ }_{\mathrm{F} p \mathrm{~L} \text { L minit }}$ \\
\hline Time to & & Trusport & He seler Checksmm & \multirow{2}{*}{\multicolumn{4}{|c|}{ Sonceeddtes }} \\
\hline \multicolumn{4}{|c|}{ Sending Address } & & & & \\
\hline \multicolumn{4}{|c|}{ Destimation Address } & \multirow{2}{*}{\multicolumn{4}{|c|}{ Destination Adtress }} \\
\hline \multicolumn{4}{|r|}{ Padding } & & & & \\
\hline
\end{tabular}

Figure 2: Schematic diagram of IPv4 and IPv6 headers

changed much between the two versions even though the new version caters for increased addresses. This has been possible since header structure has been simplified in IPv6. Some of these simplifications are: (a) options field has been eliminatedthis field was used to specify optional services like encryption, (b) header checksum field has been deleted-was used to check for errors in header, (c) service type field has been replaced with priority field- used to represent the priority of the packet and (d) time to live filed has been replaced with hop-limit field. In the new header, the flow level field has been introduced to specify a series of packets (e.g. Voice over IP) to sender or intermediate devices. Overall, the header structure in the two IP versions is distinctly different.

The experimental setup that was used to measure the performance difference of the two protocols on the operating systems is described next.

\section{EXPERIMENTAL SETUP}

Two computers with similar hardware (CPU: Intel Pentium C2D , RAM: 2GB , NIC: PCI Intel Pro 100 , Hard Drive: Seagate $160 \mathrm{~GB}$ ) were connected using a cross-over cable and each of the operating systems to be tested were installed one at a time on this test-bed (Figure 3). IPv4 as the communication protocol was configured first and data was collected. Later this was replaced with IPv6 ensuring that all other test-bed parameters remained the same. D-ITG 2.6.1d [15] was the primary tool employed to evaluate performance of protocols on operating systems. This tool was chosen because it works

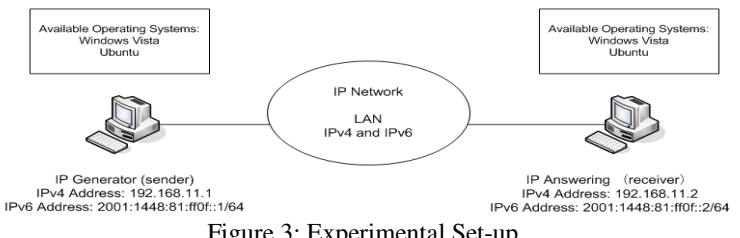

Figure 3: Experimental Set-up with both the protocol versions and the operating systems. DITG generates traffic at network/transport and application layer and sends it from sender to generator node and can measure performance related metrics.

In this research, the metrics measured were throughput, delay, jitter and CPU usage (both nodes) for TCP and UDP traffic. To ensure high data accuracy, all tests were executed 20 times, and to get the maximum throughput for a given packet size, each run had duration of 30 seconds. The results are presented and discussed next.

\section{RESUlTs}

We present the findings of the research in this section. Initially throughput values (number of bits transmissed in a certain time) for operating systems with IPv4 and IPv6 were obtained by measuring it for packet sizes ranging from 64 to 1536 Bytes. TCP results are presented in Figure 4. From this graph it is seen that for small packet sizes ( $<384$ Bytes) both operating systems with both IPv4 and IPv6 portray the same througput values, steeply increasing as the packet size increase. However, for most of the larger packet sizes Windows Vista throughput is slightly lower (average 5\%) than Linux Ubuntu. Also, IPv4 in most case give a hight throughput value than IPv6 for packet size larger than 384 Bytes.

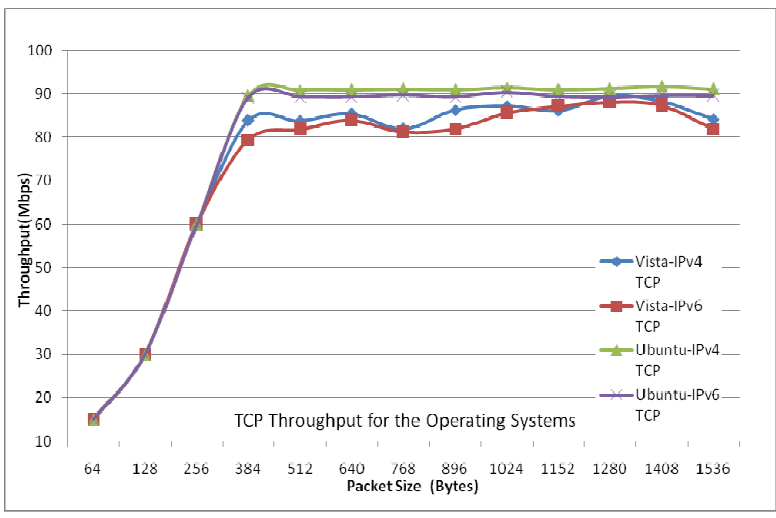

Figure 4: Throughput TCP

UDP throughput values are presented in Figure 5. Linux Ubuntu and Windows Vista mainly show a similar behavious for all packet sizes except between 384-1024 Bytes. In this range, Windows Vista again is a slightly inferior performer to its counterpart. It is worth noting that both TCP and UDP throughput values are similar for all combinations of protocols and operating systems.

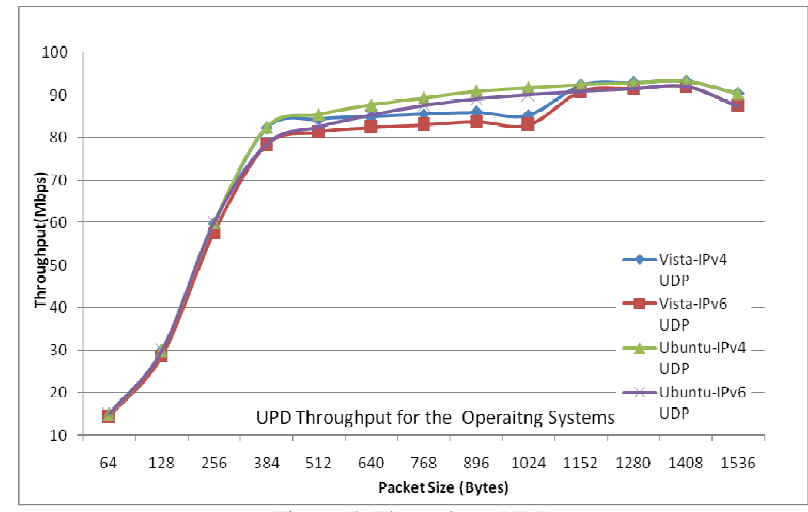

Figure 5: Throughput UDP 
Average delay for TCP and UDP traffic is shown in Figures 6 and 7 respectively. In both, there is a significant difference between the Windows and Linux distribution - the former having delay values close to zero while the latter much higher.

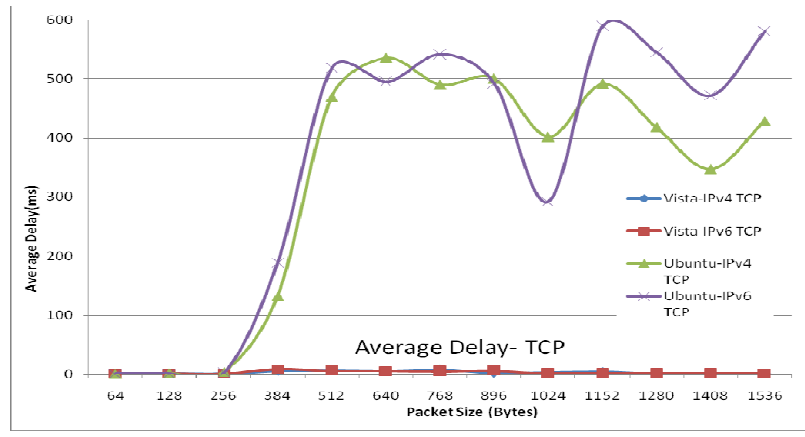

Figure 6:Average Delay TCP

Ubuntu delay values for TCP range between 300 and 600ms for both IPv4 and IPv6 and for UDP it averages around $8 \mathrm{~ms}$ for large packet sizes. For UDP, small packet sizes have low delay for all traffic types, but there is a large difference for packet sizes over 384 Bytes - Ubuntu portrays delay 4 times more than Windows Vista.

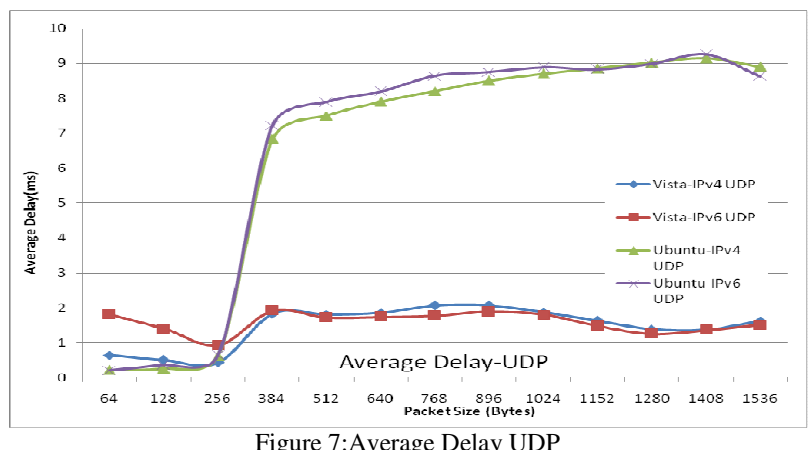

Jitter values (variation in time between packets arriving at the destination) are shown in Figures 8 and 9. For TCP there is a slight difference between the operating systems with large

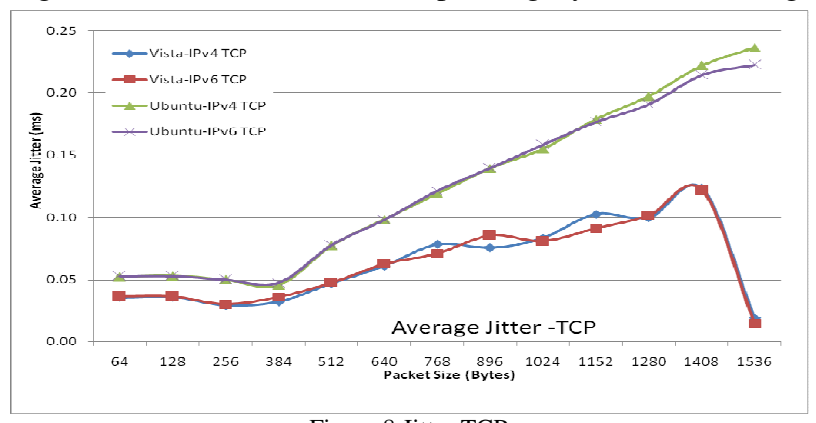

Figure 8:Jitter TCP

packet sizes however for small packets that values are very similar. In all cases Ubuntu with TCP values are higher than Windows Vista. UDP jitter shows an opposite trend (Ubuntu values are lower than Windows Vista for all packet sizes),

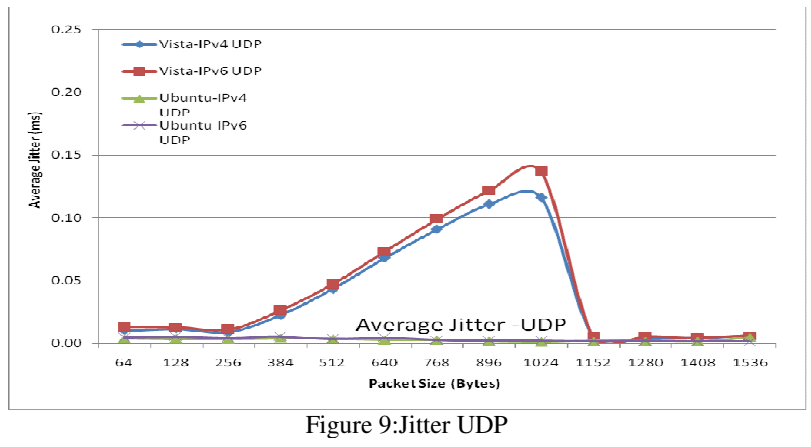

however for all packets except in the range 384-1024 Bytes, the values are similar.

CPU usage of the sender and the receiver nodes show some interesting differences between the operating systems (Figures

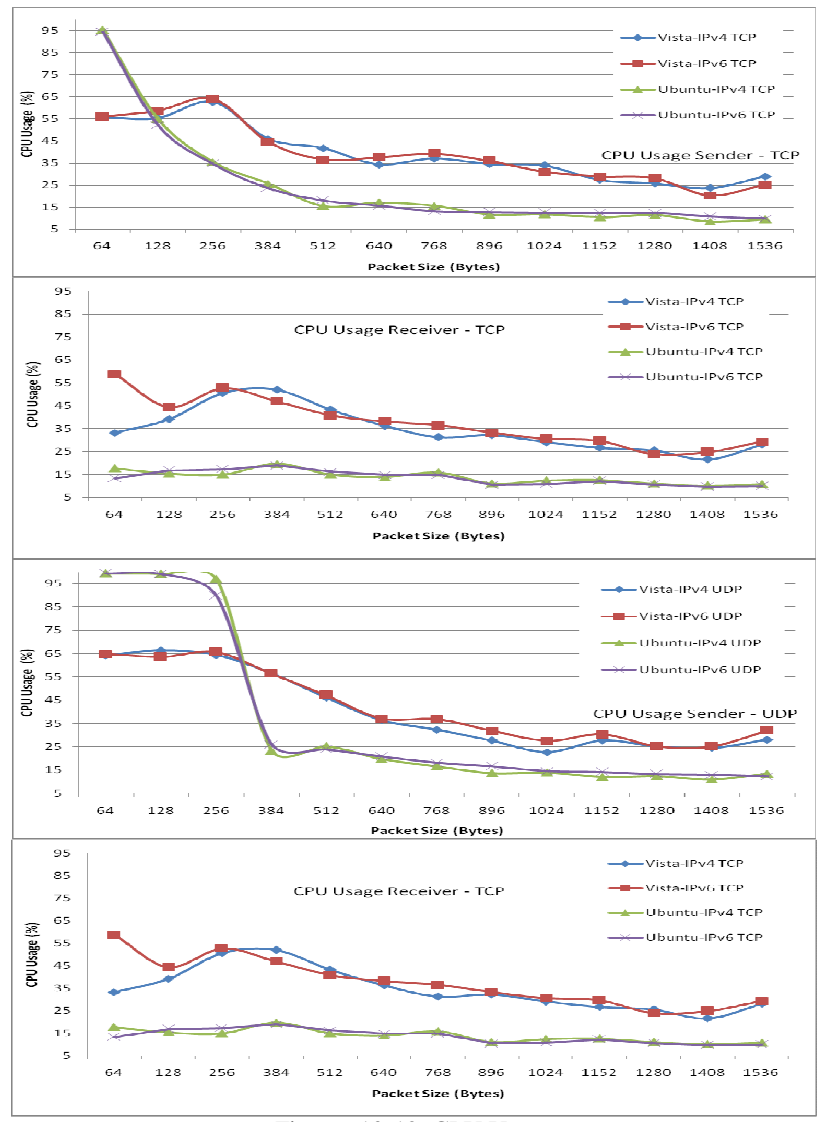

Figures 10-13: CPU Usage

10-13). In majority of the cases Windows Vista uses CPU more than Ubuntu for both TCP and UDP - in somes instances the usage is almost double. For small packet sizes Ubuntu on the sender node exceeds CPU than Windows Vista, however this can be seen as a exception.

Finally, the values for the amount of CPU utilised while decoding the received packets on the end node are presented in Figures 14 and 15. Both TCP and UDP show similar 
patterns in data and in both the cases Windows Vista uses on average $35 \%$ of CPU resouce and Ubuntu averages approximately $25 \%$ for all packet sizes.

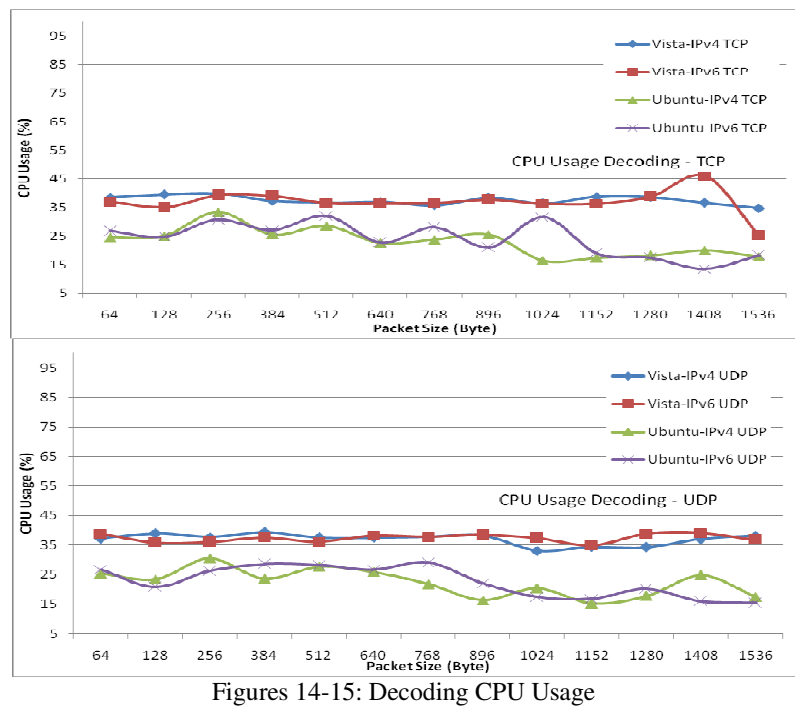

\section{CONCLUSIONS}

In this research performance of two IP versions have been empirically measured on Windows Vista and Linux Ubuntu operating systems. The following specific conclusions can be drawn:

- For packet sizes larger than 256 Bytes, IPv4 always gives a slightly better throughput than IPv6 (consistent with theory). However for small packet sizes the performance is almost identical.

- Windows Vista throughput values for most packets sizes (range: 384-1152Bytes) for both TCP and UDP traffic are lower than Linux Ubuntu by up to $5 \%$.

- There is significant difference in average delay between the operating systems. For TCP traffic, Windows Vista delay is approximately zero but Linux Ubuntu averages around $500 \mathrm{~ms}$, and for UDP Windows Vista delay is approximately 4 times lower than Ubuntu.

- Jitter values differ for the operating systems as well where Windows Vista values are lower than that of Linux Ubuntu for TCP traffic.

- For almost all packet sizes, Windows Vista uses more CPU resources on both the sending and the receiving nodes. In some situations Windows Vista uses almost double CPU resources when compared to its counterpart.

- TCP and UDP traffic decoding uses more CPU resources in Windows Vista than Linux Ubuntu.
This research has shown that the performance of IPv4 and IPv6 depends on the operating system that it has been implemented on. The extent to which performance related metrics values differ depends on the operating systems. The research team aims to extend this study to incorporate more operating systems including server environments.

\section{REFERENCES}

[1] A. Durand, "The H-Density Ratio for Address Assignment Efficiency”, IETF RFC 3194, 2001.

[2] OECD report, Internet Address Space: Economic Considerations in the Management of IPv4 and in the Deployment of IPv6, OECD Ministerial Meeting, Seoul, Korea, 17-18 June 2008.

[3] S. Narayan, S. Kolahi, Y. Sunarto, D. Nguyen, P. Mani, "Performance Comparison of IPv4 and IPv6 on Various Windows Operating Systems", Presented at the 11th IEEE International Conference on Computer and Information Technology, Khulna, 25-27 ${ }^{\text {th }}$ Dec. 2008.

[4] V. Visoottiviseth, and N. Bureenok, 'Performance Comparison of ISATAP Implementations on FreeBSD, RedHat, and Windows 2003', in Proceedings of IEEE $22^{\text {nd }}$ International Conference on Advanced Information Networking and Applications - Workshops (AINAW),Okinawa, 25-28 March 2008, pp. 547-542.

[5] S. S. Mohamed, A. Y. M. Abusin, D. Chieng, 'Evaluation of IPv6 and Comparison Study with Different Operating Systems', in Proceedings of the Information Technology and Applications 2005 (ICITA 2005), Volume 2, July 2005, pp. 665-670.

[6] S. P. Ahuja, and K. Dendukuri, 'An Empirical Evaluation of IPv6 in Linux and Windows Environments', Annual Review of Communications, Volume 59, November 2006, pp 361-370.

[7] S. Zeadally, R. Wasseem, and I. Raicu, 'Comparison of Endsystem IPv6 Protocol Stack', in Proceedings of IEE Internet Protocols, Technology and Applications (VoIP), Volume 151, Issue 3, 25 June 2004, pp 238 - 242.

[8] S. Zeadally, and I. Raicu, 'Evaluating IPv6 on Windows and Solaris', IEEE Internet Computing, Volume 7, Issue 3, MayJune 2003, pp 51-57.

[9] M. Anand, Netperf3 TCP Network Performance on IPv6 using 2.4.17 Kernel. IBMLinux Technology Center, www124.ibm.com/developworks/opensource/linuxperf/netperf/results /may_02/netperf3_ipv6_2.4.17 results.htm.

[10] K. K. Ettikan, 'IPv6 Dual stack Transition Technique Performance Analysis: KAME on FreeBSD as the Case'. Faculty of Information Technology, Multimedia University, Jalan Multimedia, October 2000.

[11] S. Ariga, K. Nagahashi, M. Minami, H. Esaki, J. Murai, 'Performance Evaluation of Data Transmission Using IPSec over IPv6 Networks', INET 2000 Proceedings, Japan, July $18^{\text {th }}$, 2000.

[12] P. P. Xie, Network Protocol Performance Evaluation of IPv6 for Windows NT. Masters thesis, California Polytechnic State University, San Luis Obispo, June 1999.

[13] R. P. Draves, A. Mankin, and B.D. Zill, 'Implementing IPv6 for Windows NT'. In the Proceedings of the $2^{\text {nd }}$ USENIX Windows NT Symposium, Seattle, WA, USA, August 1998.K. Elissa, "Title of paper if known," unpublished.

[14] M. Tufail, "IP v6 - An opportunity for new service and network features", IEEE International conference on Networking and Services (ICNS), July 2006, Silicon Valley, USA.

[15] A. Botta, A. Dainotti, A. Pescapè, "Multi-protocol and multiplatform traffic generation and measurement", INFOCOM 2007 DEMO Session, May 2007, Anchorage (Alaska, USA). 